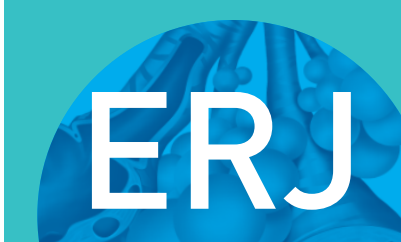

open research

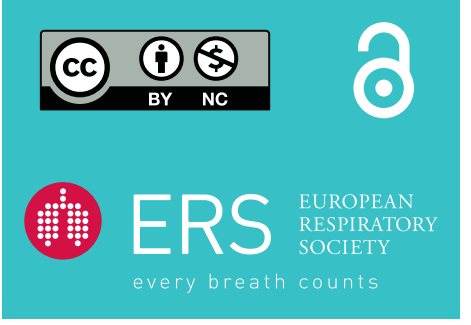

\section{Subtle immunodeficiencies in nodular-bronchiectatic Mycobacterium avium complex lung disease}

\author{
To the Editor:
}

Mycobacterium avium complex (MAC) bacteria are the most frequent causative agents of nontuberculous mycobacterial (NTM) diseases worldwide. The most frequent disease manifestation is MAC lung disease (MAC-LD), which can present as tuberculosis-like fibro-cavitary disease or as a nodular-bronchiectatic disease. The fibro-cavitary disease is associated with COPD and inhaled steroid use, but the predisposing conditions for nodular-bronchiectatic MAC-LD are not fully understood [1]. The current dogma is that it results from a multisystem, multigenic disorder as evidenced by whole exome sequencing studies [2]. Immunodeficiencies have been specifically studied in this context, and 35\% of pulmonary NTM disease had variants in immune pathway genes including STAT1, IRF8, CARD9, CLEC4D and MPEG1 [2]. The cytokine production profiles of patients with MAC-LD have not been well studied. There is evidence for a protective role for interferon (IFN)- $\gamma$, interleukin (IL)-12, CXCL10 and IL-17, while IL-10 is immunosuppressive [3,4]. In this case-series, a cohort of 13 female nodular-bronchiectatic MAC-LD patients (NB-MAC-LD) underwent peripheral blood mononuclear cells (PBMCs) cytokine production testing, in response to (myco)bacterial and fungal antigens as well as recombinant cytokines. To evaluate the immune function, we compared cytokine production profiles between patients and healthy controls.

The 13 patients had a mean \pm SD age of $66 \pm 8.4$ years; 11 patients were being treated at the time of immunological testing, while two patients were receiving hypertonic saline inhalation without antibiotics due to intolerance. $5 \times 10^{5}$ PBMCs of the 13 patients and nine female healthy controls were isolated as previously described [5] and stimulated with 1) several NTM species $\left(1 \times 10^{6}\right.$ cells $\cdot \mathrm{mL}^{-1}$ [6]): the patient's own mycobacterial strain ("M. avium patient”), M. avium ATCC 700898 ("M. avium ref."), Mycobacterium abscessus CIP 104536 ("M. abscessus ref."), Aspergillus fumigatus VO5 $\left(1 \times 10^{7}\right.$ conidia.mL $\left.\mathrm{mL}^{-1}\right)$, Candida albicans UC820 $\left(1 \times 10^{6}\right.$ conidia. $\left.\mathrm{mL}^{-1}\right)$, Staphylococcus aureus ATCC $29213\left(1 \times 10^{6} \mathrm{U} \cdot \mathrm{mL}^{-1}\right)$ and Mycobacterium tuberculosis (MTB) sonicated H37Rv $\left(1 \mu \mathrm{g} \cdot \mathrm{mL}^{-1}\right)$; and 2) a set of ligands and polarising cytokines: IL-1 $\alpha \quad\left(10 \mathrm{ng} \cdot \mathrm{mL}^{-1}\right), \quad$ IL-1 $\beta \quad\left(10 \mathrm{ng} \cdot \mathrm{mL}^{-1}\right), \quad$ IL-12 $\quad\left(10 \mathrm{ng} \cdot \mathrm{mL}^{-1}\right), \quad$ IL-23 $\quad\left(50 \mathrm{ng} \cdot \mathrm{mL}^{-1}\right)$, phytohaemoagglutinin (PHA; $10 \mu \mathrm{g} \cdot \mathrm{mL}^{-1}$ ), Escherichia coli lipopolysaccharide (LPS; $10 \mathrm{ng} \cdot \mathrm{mL}^{-1}$ ), LPS with IFN- $\gamma\left(1 \mu \mathrm{g} \cdot \mathrm{mL}^{-1}\right)$, LPS with IL-12, Pam3Cys $\left(10 \mu \mathrm{g} \cdot \mathrm{mL}^{-1}\right)$ and Pam3Cys with $\beta$-glucan $\left(10 \mu \mathrm{g} \cdot \mathrm{mL}^{-1}\right)$ for either $24 \mathrm{~h}$ or 7 days (in the presence of $10 \%$ human serum [6]) at $37^{\circ} \mathrm{C}$; and 3 ) with RPMI, as a negative control. The innate cytokines interleukin IL-6 and IL-1 $\beta$ were measured after $24 \mathrm{~h}$ and the adaptive cytokines interferon IFN- $\gamma$, IL-10, IL-17 and IL-22 were measured using ELISA after 7 days in the cell culture supernatant.

In the innate immune responses, NB-MAC-LD patients did not demonstrate defects in pro-inflammatory cytokine production after PBMC stimulation for $24 \mathrm{~h}$ with any stimulus (figure 1a). Interestingly, NB-MAC-LD patients showed a significantly higher anti-inflammatory IL-1Ra production on M. avium and M. abscessus stimulation compared with the control group (figure 1b). Since Dectin-1 functions together with toll-like receptor (TLR)2 [7] and IFN- $\gamma$ boosts TLR4 signalling [8] to mediate mycobacterial-induced innate cytokine production, functional synergisms of those receptors through their ligands have been checked, but no defective signalling in patients was detected (figure 1c).

@ERSpublications

Patients with nodular-bronchiectatic MAC lung disease have dysregulated adaptive immunity with defective IL-17 and IFN- $\gamma$ production, and IL-10 overproduction. This suggests a role for adjunctive immunomodulatory treatments. https://bit.ly/33AALwx

Cite this article as: Bruno M, Zweijpfenning $\mathrm{SMH}$, Verhoeven J, et al. Subtle immunodeficiencies in nodular-bronchiectatic Mycobacterium avium complex lung disease. ERJ Open Res 2020; 6: 005482020 [https://doi.org/10.1183/23120541.00548-2020]. 


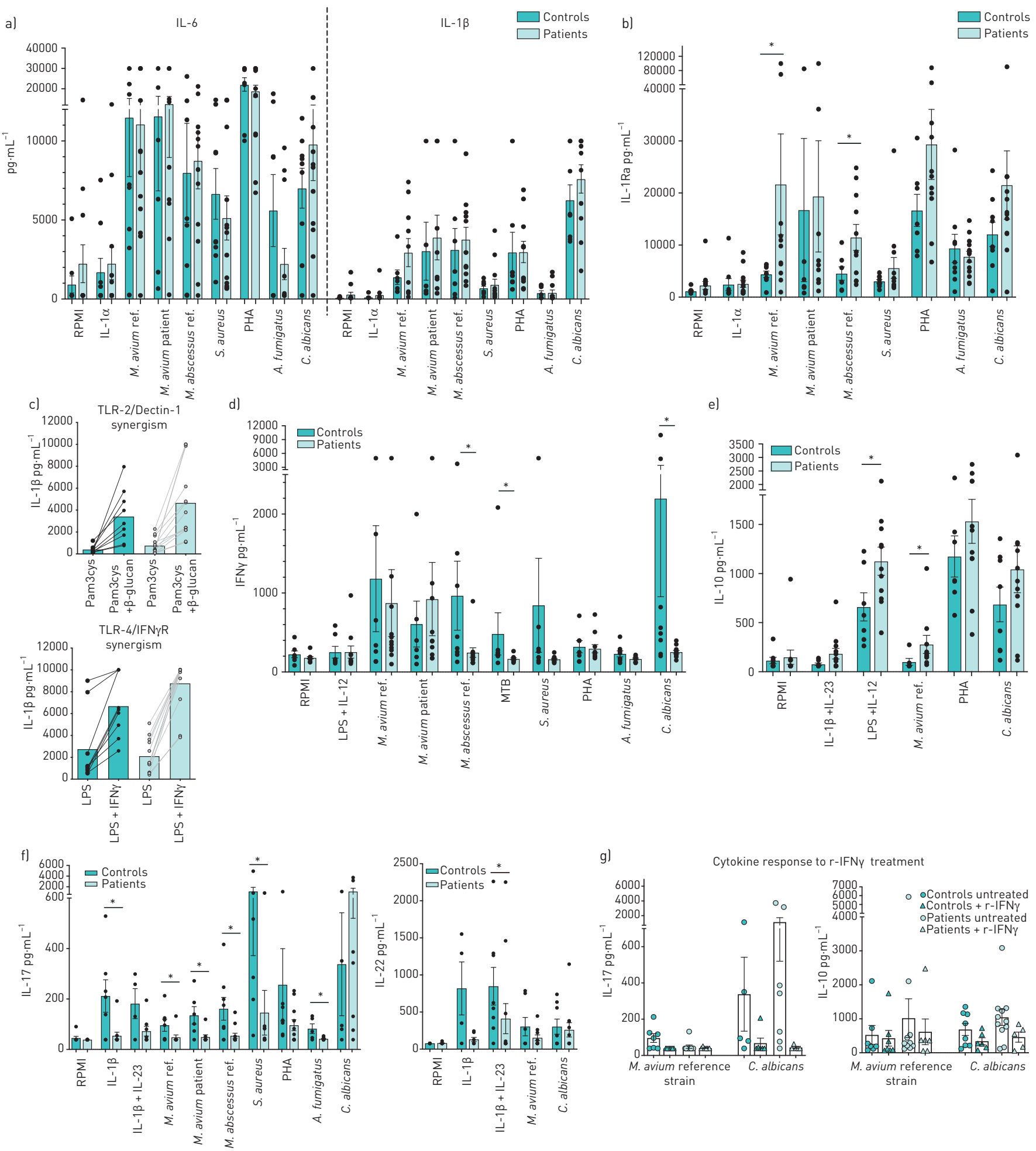

FIGURE 1 Innate and adaptive cytokine profile in nodular-bronchiectatic Mycobacterium avium complex-lung disease patients and controls. a-c) Interleukin (IL)-6, IL-1 $\beta$ and IL-1Ra production after $24 \mathrm{~h}$ in patients ( $\mathrm{n}=13$ ) and controls ( $\mathrm{n}=9$ ) under different stimulation conditions. d) Interferon (IFN)- $\gamma$, e) IL-10, f) IL-17 and IL-22 after $24 \mathrm{~h}$ in patients ( $\mathrm{n}=12)$ and controls $(\mathrm{n}=8)$ upon microbial stimuli and polarising cytokines. g) IL-17 and IL-10 production after 7 days in response to $M$. avium ref. and $C$. albicans with or without $1 \mathrm{~h}$ pre-incubation with IFN- $\gamma\left(1 \mu \mathrm{g} \cdot \mathrm{mL}^{-1}\right.$ ). Data are shown as mean \pm SEM. Significance was determined using the Mann-Whitney U-test. *: p-value $<0.05$, this was considered statistically significant. M. avium ref.: M. avium ATCC 700898; M. avium patient: the patient's own M. avium strain; M. abscessus ref.: Mycobacterium abscessus CIP 104536; S. aureus: Staphylococcus aureus ATCC 29213; PHA: phytohaemoagglutinin; A. fumigatus: Aspergillus fumigatus V05; C. albicans: Candida albicans UC820; TLR: toll-like receptor; LPS: lipopolysaccharide; MTB: Mycobacterium tuberculosis: r-IFN- $\gamma$ : recombinant IFN- $\gamma$. 
The adaptive immune response revealed an imbalance between T-helper (Th)1, Th17/22 and regulatory T-cells, with the predominance of the latter. No significant differences in IFN- $\gamma$ release in response to $M$. avium were observed between the two groups; however, IFN- $\gamma$ production was significantly reduced in patients in response to M. abscessus, M. tuberculosis, and C. albicans (figure 1d). A trend towards higher IL-10 production, a cytokine with potent anti-inflammatory properties, across a range of stimuli was found in patients as compared with controls; in particular, a significant increase upon stimulation with the Th1 polarising ligands LPS+IL-12 and M. avium was observed (figure 1e). IL-17 and IL-22 levels were generally lower in patients upon nearly all the stimulations, with a significant difference in IL-17 between patients and controls upon the polarising cytokine IL-1 $\beta$, M. avium (reference and patient strain), M. abscessus, S. aureus and A. fumigatus. Also, the combination of polarising Th17/22 cytokines IL-23+ IL-1 $\beta$ led to significantly lower IL-22 production in patients. Only C. albicans (used as a positive control for IL-17 production) triggered a trend for higher IL-17 production in patients compared with controls (figure 1f). The fact that the lymphocytes do not polarise properly might lead to defective Th1 and Th17 function, and an excess of regulatory T-cells that impairs effective eradication of NTM with subsequent persistence or recurrence of infection.

Treatment with recombinant IFN- $\gamma$ downregulates $M$. avium-induced IL-17 in healthy controls, but not in the patients, whose IL-17 production is already very low upon this stimulation. Interestingly, in vitro pre-treatment with recombinant IFN- $\gamma$ reduced C. albicans and M. avium-induced IL-10 production in patients, bringing them to levels similar to the healthy controls (figure 1g). Previous clinical studies have demonstrated mixed results with the use of recombinant IFN- $\gamma$ in MAC-LD patients $[9,10]$. Interestingly, the anti-inflammatory cytokines IL-1Ra and IL-10 were significantly increased also in patients with fibro-cavitary NTM-LD disease [4]. One might speculate that the higher IL-1Ra production contributes to the decreased IL-1 $\beta$ response which has been reported in NTM-LD patients [11], and that the excessive IL-10 production, most likely Th2 and T-regs derived [12], might interfere with polarisation toward Th1/Th17 lymphocytes and with proper alveolar macrophage activation. In fact, IL-10 has been described to limit IFN- $\gamma$ and IL-17 responses during bacille Calmette-Guérin (BCG) vaccination in mice [13].

A defect in Th17 immunity that we found in our cohort has been also described in other studies: WU et al. [14] reported significantly reduced proportions of IL-17A producing CD4 ${ }^{+}$T-cells and IL-17 cytokine levels in NTM-LD patients; BECKER et al. [15] reported a reduction in M. abscessus-induced IL-17 production in cystic fibrosis patients with M. abscessus-LD and, in line with our data, they also observed a normal IL-17 production in response to C. albicans in patients; this phenomenon is possibly related to the fact that the Candida cell wall contains $\beta$-glucans, which are recognised by the Dectin- 1 receptor, whose signalling was intact in our patients.

The observed cytokine production suggests specific immunodeficiencies, predisposing these patients to MAC-LD, perhaps as part of a wider multisystem disorder [2]. This re-opens the debate on the role of adjunctive immunomodulatory treatments to improve the outcomes of MAC-LD. However, IFN- $\gamma$ trials showed mixed results $[9,10]$; perhaps stricter enrolment criteria, based on cytokine production testing or genetic testing, can identify patients who are most likely to benefit from adjunctive immunomodulatory treatments, where recombinant IFN- $\gamma$ would be one of the options. Our results suggest that NB-MAC-LD disease may be associated with cellular responses that favour production of anti-inflammatory cytokines, such as IL-10. Neutralising IL-10 production or related-signalling pathways might be a therapeutic strategy for the control of MAC-LD disease. It has been already proven that anti-IL-10 treatment reduced the bacterial load in M. avium-infected mice [16] and conferred higher protection against M. tuberculosis through sustained Th1 and Th17 responses [17]. Also, the stimulus-specific deficient IL-17 production in NTM-LD patients may provide a rationale for adjunctive supplementation of low dose-recombinant IL-17. The immune signature we found in this exploratory study is very consistent and this represent a major advance to an understudied disease. However, the authors acknowledge the limitations of the current study, such as the small cohort, as well as the fact that we were not able to perform a very extensive immune profiling. This hampers drawing firm conclusions from the data presented.

In conclusion, patients with nodular-bronchiectatic MAC-LD from our cohort showed a dysregulated adaptive immunity, with a defective IL-17 and IFN- $\gamma$ production and an exaggerated IL-10 production, which was re-balanced upon pre-treatment with recombinant IFN- $\gamma$. These immunodeficiencies add to the "multisystem, multigenic" dogma. By contrast, patients did not present a defective pro-inflammatory innate immune response, as evidenced by normal IL- 6 or IL- $1 \beta$ levels, but it is tempting to speculate that the significantly higher anti-inflammatory IL-1Ra production in response to NTM would interfere with an effective bacterial eradication. The observed immunodeficiencies suggest a role for novel adjunctive immunomodulatory treatments to improve the outcomes of MAC-LD. 
Mariolina Bruno $\oplus^{1}$, Sanne M.H. Zweijpfenning ${ }^{2}$, Jeske Verhoeven ${ }^{2}$, Martin J. Boeree ${ }^{2}$, Mihai G. Netea ${ }^{1,3}$, Frank L. van de Veerdonk ${ }^{1}$, Jakko van Ingen $\oplus^{4}$ and Wouter Hoefsloot $\oplus^{2}$

${ }^{1}$ Dept of Internal Medicine and Radboud Center for Infectious Diseases, Radboud University Medical Center, Nijmegen, The Netherlands. ${ }^{2}$ Radboudumc Center for Infectious Diseases, Dept of Pulmonary Diseases, Radboud University Medical Center, Nijmegen, The Netherlands. ${ }^{3}$ Dept for Genomics and Immunoregulation, Life and Medical Sciences Institute (LIMES), University of Bonn, Bonn, Germany. ${ }^{4}$ Radboudumc Center for Infectious Diseases, Dept of Medical Microbiology, Radboud University Medical Center, Nijmegen, The Netherlands.

Correspondence: Wouter Hoefsloot, Radboud University Medical Centre, Dept of Pulmonary Diseases, PO box 9101, 6500HB Nijmegen, The Netherlands. E-mail: Wouter.Hoefsloot@radboudumc.nl

Received: 2 Aug 2020 | Accepted: 3 Aug 2020

Acknowledgement: The authors thank Lian J. Pennings (Dept of Medical Microbiology, Radboud University Medical Centre, Nijmegen, The Netherlands) for logistic support in the laboratory and providing the mycobacterial strains, and D. Rosati (Dept of Internal Medicine, Radboud University Medical Centre, Nijmegen, The Netherlands) for the help with the ELISA assays.

Conflict of interest: M. Bruno has nothing to disclose. S.M.H. Zweijpfenning reports grants and nonfinancial support from Insmed and Novartis outside the submitted work. J. Verhoeven has nothing to disclose. M.J. Boeree has nothing to disclose. M.G. Netea has nothing to disclose. F.L. van de Veerdonk has nothing to disclose. J. van Ingen has nothing to disclose. W. Hoefsloot has nothing to disclose.

Support statement: F.L. van de Veerdonk was supported by a Vidi grant from the Netherlands Organisation for Scientific Research and a Nijmegen Centre for Molecular Life Sciences grant from Radboud University Nijmegen Medical Centre. M.G. Netea was supported by an ERC Advanced Grant (number 833247) and a Spinoza Grant from the Netherlands Organisation for Scientific Research. J. van Ingen was supported by a Veni grant from the Netherlands Organisation for Scientific Research (number 016.176.024).

\section{References}

1 Cowman S, van Ingen J, Griffith DE, et al. Non-tuberculous mycobacterial pulmonary disease. Eur Respir J 2019; 54: 1900250 .

2 Szymanski EP, Leung JM, Fowler CJ, et al. Pulmonary nontuberculous mycobacterial infection. A multisystem, multigenic disease. Am J Respir Crit Care Med 2015; 192: 618-628.

3 Vankayalapati R, Wizel B, Samten B, et al. Cytokine profiles in immunocompetent persons infected with Mycobacterium avium complex. J Infect Dis Oxford Academic 2001; 183: 478-484.

4 Bamba $\mathrm{Y}$, Moro $\mathrm{H}$, Aoki N, et al. Multiplex cytokine analysis in Mycobacterium avium complex lung disease: relationship between CXCL10 and poor prognostic factors. BMC Infect Dis 2019; 19: 263.

5 Smeekens SP, Ng A, Kumar V, et al. Functional genomics identifies type I interferon pathway as central for host defense against Candida albicans. Nat Commun 2013; 4: 1342.

6 Surolia R, Karki S, Wang Z, et al. Attenuated heme oxygenase-1 responses predispose the elderly to pulmonary nontuberculous mycobacterial infections. Am J Physiol Lung Cell Mol Physiol 2016; 311: L928-L940.

7 Yadav M, Schorey JS. The $\beta$-glucan receptor dectin-1 functions together with TLR2 to mediate macrophage activation by mycobacteria. Blood 2006; 108: 3168-3175.

8 Heldwein KA, Liang MD, Andresen TK, et al. TLR2 and TLR4 serve distinct roles in the host immune response against Mycobacterium bovis BCG. J Leukoc Biol 2003; 74: 277-286.

9 Milanés-Virelles MT, García-García I, Santos-Herrera Y, et al. Adjuvant interferon gamma in patients with pulmonary atypical Mycobacteriosis: A randomized, double-blind, placebo-controlled study. BMC Infect Dis 2008; 8: 17.

10 Lam PK, Griffith DE, Aksamit TR, et al. Factors related to response to intermittent treatment of Mycobacterium avium complex lung disease. Am J Respir Crit Care Med 2006; 173: 1283-1289.

11 Wu M-F, Shu C-C, Wang J-Y, et al. NLRP3 inflammasome is attenuated in patients with Mycobacterium avium complex lung disease and correlated with decreased interleukin-1 $\beta$ response and host susceptibility. Sci Rep 2019; 9: $1-10$.

12 Moreira-Teixeira L, Redford PS, Stavropoulos E, et al. T cell-derived IL-10 impairs host resistance to Mycobacterium tuberculosis infection. J Immunol 2017; 199: 613-623.

13 Gopal R, Lin Y, Obermajer N, et al. IL-23-dependent IL-17 drives Th1-cell responses following Mycobacterium bovis BCG vaccination. Eur J Immunol 2012; 42: 364-373.

14 Wu U-I, Olivier KN, Kuhns DB, et al. Patients with idiopathic pulmonary nontuberculous mycobacterial disease have normal Th1/Th2 cytokine responses but diminished Th17 cytokine and enhanced granulocyte-macrophage colony-stimulating factor production. Open Forum Infect Dis 2019; 6: ofz484.

15 Becker KL, van Ingen J, ten Oever J, et al. Deficient interleukin-17 production in response to Mycobacterium abscessus in cystic fibrosis. Eur Respir J 2016; 47: 990-993.

16 Bermudez LE, Champsi J. Infection with Mycobacterium avium induces production of interleukin-10 (IL-10), and administration of anti-IL-10 antibody is associated with enhanced resistance to infection in mice. Infect Immun 1993; 61: 3093-3097.

17 Pitt JM, Stavropoulos E, Redford PS, et al. Blockade of IL-10 signaling during BCG vaccination enhances and sustains Th1, Th17, and innate lymphoid IFN- $\gamma$ and IL-17 responses and increases protection to Mycobacterium tuberculosis infection. J Immunol 2012; 189: 4079-4087. 